\title{
Kolorektales Karzinom - Standard und neue Wege
}

\author{
Gesprächsleiter: Hans Jürgen Schlitt (Regensburg) Thomas Seufferlein (Halle/Saale) \\ Pompiliu Piso (Regensburg)
}

Teilnehmer: $\quad$ Dirk Arnold (Hamburg) Hauke Lang (Mainz)

Anke Reinacher-Schick (Bochum)

Frage 1: Welche Behandlungsmöglichkeiten ergeben sich aus onkologischer Sicht bei Peritonealkarzinose (bei kolorektalen Karzinomen (KRK))?

Lang: Die chirurgische Standardtherapie der Peritonealkarzinose beim KRK konzentrierte sich bislang auf die Sicherstellung der Darmpassage im Falle manifester oder drohender Komplikationen. Für ein hoch selektioniertes Patientenkollektiv mit einer rein peritonealen, intrakavitären Metastasierung rückt die Peritonektomie als «zytoreduktive Chirurgie» (CRS) mit intraoperativer hyperthermer intraperitonealer Chemoperfusion (HIPEC) zunehmend in das Blickfeld der Chirurgie. In der Literatur ist für dieses Verfahren, in überwiegend retrospektiven Analysen, eine Verdoppelung des tumorfreien Überlebens gegenüber einer systemischen Standardtherapie bei zudem verbesserter Lebensqualität beschrieben. Prospektiv randomisierte Daten der CRS/HIPEC im Vergleich mit einer modernen palliativen Chemotherapie müssen allerdings noch erarbeitet werden.

Arnold: Bei einer lokal begrenzten Peritonealkarzinose kann sich, beim optimalen Zusammenspiel der verschiedenen Disziplinen, aus der Kombination systemische Chemotherapie, CRS und möglicherweise auch HIPEC ein kurativer Ansatz ergeben. Dennoch: Dies wird nur für die wenigsten Patienten zutreffen. Für die meisten Patienten ist auch bei ausschließlich peritonealer Manifestation die systemische Chemotherapie die Therapie der Wahl.

Reinacher-Schick: Systemische Chemotherapie je nach Vortherapie und Begleiterkrankungen; bei EPCAM-positiver Erkrankung Catumaxomab, falls austherapiert; bei sonst resektabler Erkrankung: Peritonektomie und HIPEC in einem ausgewiesenen Zentrum.

\section{KARGER}

Fax +497614520714

Information@Karger.de

www.karger.com (c) 2011 S. Karger GmbH, Freiburg

Accessible online at:

www.karger.com/vim
Frage 2: Warum soll ein Rektumkarzinom nicht laparoskopisch operiert werden?

Lang: Die Ergebnisse der Rektumchirurgie haben sich durch die Einführung der totalen mesorektalen Exzision (TME) dramatisch verbessert. Nachdem initiale Fallberichte und tierexperimentelle Untersuchungen ein erhöhtes Risiko einer Tumorzellverschleppung durch die laparoskopische Chirurgie suggeriert haben, konnten mehrere randomisierte Studien keinen tumorspezifischen Nachteil der laparoskopischen Resektion gegenüber der offenen Technik belegen. Sofern die onkochirurgischen Prinzipien der Rektumresektion eingehalten werden, stellt die laparoskopische Resektion in der Hand des erfahrenen, laparoskopisch versierten Kolorektalchirurgen somit ein mindestens gleichwertiges Verfahren dar. Der Nachweis eines onkologischen Vorteils der laparoskopischen gegenüber der konventionellen Chirurgie konnte bislang nicht geführt werden. Ausgesprochen problematisch bleibt die intraoperative Konversion, die eindeutig als negativer onkologischer Prognosefaktor identifiziert werden konnte.

Arnold: Bei prinzipiell chirurgischer Gleichwertigkeit des laparoskopischen gegenüber des «offenen»Verfahrens beim Kolonkarzinom bestehen beim Rektumkarzinom doch noch deutliche Unterschiede, insbesondere hinsichtlich der Expertise der Zentren. Allerdings kann offenbar in «high volume»Zentren auch eine laparoskopische Rektumresektion onkologisch gleichwertig werden.

Reinacher-Schick: Dies ist eine chirurgische Frage. Falls onkologisch gleichwertige Ergebnisse vorliegen, dann sollte es der Erfahrung und Entscheidung des Operateurs überlassen sein, wie er operiert. 
Frage 3: Welche Vorteile hat die Einführung zertifizierter Darmzentren aus Ihrer Sicht mit sich gebracht?

Lang: Die Ergebnisse der Therapie des (metastasierten) KRK sind insbesondere den verbesserten multimodalen Therapiekonzepten zuzuschreiben. Die Zertifizierung leitet die Patienten (und ihre Hausärzte) in die Zentren, die die notwendige Infrastruktur für die komplexe Therapie dieser Tumorerkrankungen vorhalten. Die Zertifizierung macht diese Zentren nach außen erkennbar, und durch die regelmäßige Rezertifizierung wird zudem dauerhaft eine personenunabhängige, hohe Prozessqualität gewährleistet. Durch die Zertifizierungsvorgaben ist nicht nur die Behandlung des kolorektalen Primarius flächendeckend standardisiert, sondern die verpflichtende Kooperation kleinerer Häuser mit entsprechenden Kompetenzzentren für die Therapie z.B. von Lebermetastasen sorgt auch für eine umfassende, standardisierte Patientenversorgung auf einem flächendeckend hohen Niveau.

Arnold: Sicher hat die Einführung der Standards für Darmkrebszentren in diesen einen erheblichen Qualitätsschub, gerade hinsichtlich Benchmarking und Qualitätssicherung, gebracht. Allerdings werden viele wichtige Kriterien auch nicht erfasst bzw. das Benchmarking führt anhand der genannten Kriterien mitunter auch zu einer qualitativen Fehlentwicklung - vor allem, wenn nur die Erfüllung der Zahlen im Vordergrund steht.

Reinacher-Schick: Verbesserung der Qualität onkologischer Versorgung, Implementierung von Leitlinien, flächendeckende Verbesserung der Versorgung sowie Unterstützung von Studienaktivitäten. Hinzu kommt eine Stärkung der Interdisziplinarität in Tumorboards (gefordert).

Frage 4: Kommt die Beurteilung der Lebensqualität in der Erstellung von Therapiekonzepten zu kurz?

Lang: Die Beurteilung der Lebensqualität hat in der Therapie von Tumorpatienten höchste Priorität. Insbesondere bei Patienten mit unheilbaren Tumorstadien müssen der Therapiewunsch und die Therapieoptionen mit der zu erwartenden Lebensqualität abgewogen werden. Am Beispiel der Darmzentren mit den obligatorisch integrierten Konzepten zur standardisierten Schmerztherapie, Stomatherapie und psychoonkologischen Betreuung wird das geschärfte Bewusstsein für die Bedeutung der Lebensqualität widergespiegelt. Lebensqualität gewinnt insbesondere vor dem Hintergrund langer Krankheitsverläufe unter optimierter moderner Palliativtherapie an Bedeutung. Nicht zuletzt die Darmzentren tragen diesem Umstand Rechnung. In vielen Bereichen der Chirurgie ist ein Trend zu minimal invasiven Verfahren zu verzeichnen, was nicht zuletzt einer verbesserten Lebensqualität des Patienten zugute kommt.
Arnold: In jedem Fall sollte in Zukunft bei der Entwicklung von Therapieverfahren beim metastasierten KRK mehr Wert auf die Evaluation von Lebensqualität gelegt werden: Es ist prinzipiell klar, dass sich z.B. in der metastasierten Situation Ansprechen, Rückgang, Verlängerung des progressionsfreien Intervalls und Lebensqualität parallel entwickeln - d.h., auch die Lebensqualität bleibt länger erhalten oder bessert sich (bei symptomatischen Patienten). Allerdings müssen moderne Konzepte, die einerseits auf eine Therapieintensivierung setzen, und andere, die auf eine Therapieunterbrechung oder -deeskalation zielen, hierin besonders sorgfältig begleitet werden.

Reinacher-Schick: Ja. Noch immer. Diese sollte im Hinblick auf das Arzneimittelmarktneuordnungsgesetz (AMNOG) einen höheren Stellenwert bekommen.

Frage 5: Nennen Sie Ihre wichtigsten Kriterien für eine «personalisierte» Therapie bei Patienten mit KRK.

Lang: Therapiewunsch: Die Intensität und Dauer einer Therapie sollten auf die Bedürfnisse des einzelnen Patienten abgestimmt werden. Allgemeinzustand: Der Allgemeinzustand des Patienten hat großen Einfluss auf die zur Auswahl stehenden Therapieoptionen. Tumorcharakteristika (Biomarker?): Lymphknotenstatus und K-ras-Status des Primarius sind ebenso wichtig wie die Dynamik der individuellen Tumorerkrankung und viele weitere Faktoren.

Arnold: «Personalisierte Medizin» wird auch in Zukunft verschiedene Dimensionen umfassen: Krankheitscharakteristika, klinische Patientencharakteristika und die wichtige Frage der Patientenmotivation - und molekulare Charakteristika in prognostischer und prädiktiver Hinsicht. Wünschenswert wäre, wenn weitere Biomarker vor allem in prädiktiver Hinsicht vor allem für den Nutzen einer Therapie entwickelt werden könnten. Dennoch können wir sicher davon ausgehen, dass auch weiterhin die eher «klassischen» Parameter wie Patientenund Therapiecharakteristika und deren Zielsetzung eine Rolle spielen werden.

Reinacher-Schick: R0-Resektion/Kuration möglich? Tumorbedingte Symptome? Frühes Ansprechen als Prognosefaktor; Alter; K-ras-Status; Mikrosatelliteninstabilität (MSI) bei Niedrigrisiko-Stadium-II-Karzinomen.

Frage 6: Wie können wir die Vielzahl neuer Substanzen in die Therapie des KRK integrieren und wie gehen wir mit der länger werdenden Therapiedauer in der palliativen Situation um?

Lang: Die zunehmenden Therapieoptionen und somit die zunehmende Therapiedauer bedürfen höchster Kompetenz auf 
dem Gebiet der gastrointestinalen Onkologie. Der Einsatz neuer Substanzen in der Standardtherapie des KRK setzt jedoch die Testung in randomisierten Studien voraus. Die Infrastruktur zur Durchführung und zur Teilnahme an klinischen Studien muss weiter gestärkt und professionalisiert werden. Die Beibehaltung hoher wissenschaftlicher und ethischer Standards in der Durchführung klinischer Prüfungen muss dennoch mit einem niederschwelligen Angebot auch an außeruniversitäre Einrichtungen zur Teilnahme verbunden sein. Die Formulierung und regelmäßige Aktualisierung von Leitlinien, wie sie seit einiger Zeit unter anderem als S3-Leitlinie für das KRK vorliegen, sind ein essenzieller Bestandteil der flächendeckenden Qualitätssicherung und Implementierung neuer Verfahren und Substanzen.

Die sich ergebende längere Therapiedauer durch höhere Effektivität ermöglicht zudem auch chirurgische Optionen im Verlauf der Therapie, die dann unbedingt regelmäßig reevaluiert und wahrgenommen werden sollten.

Arnold: Ganz sicher ist, dass wir nicht «zu viele» neue Substanzen haben: Bei der bisherigen «Erfolgsrate» der Entwicklung - d.h. einer wirklichen und relevanten Prognoseverbesserung bei nur 20 bis maximal 30\% der Behandelten - brauchen wir eher mehr Substanzen, um alle Subsegmente von Patienten abzudecken. Insofern wird die weitere Entwicklung vor allem in kleineren Kollektiven - und kleineren Studien stattfinden; die Kollektive sind dabei nach molekularen Parametern stratifiziert. Die neue Studienplattform der European Organisation for Research and Treatment of Cancer (EORTC) ist hier ein gutes Beispiel, wie die molekulare Pathologie und entsprechende klinische Studien gut zusammengebracht werden.

Auch hinsichtlich der Therapie besteht großes Entwicklungspotenzial: Die Kombinationsschemata mit (potenziell) symptomatischer Toxizität werden im Regelfall immer kürzer durchgeführt - hingegen kommen nun Strategien mit Erhaltungstherapien bzw. Deeskalation in die Klinik. Hier sind gegenwärtig schon erste Substanzen in Entwicklung, die nur während der Erhaltungstherapiephase eingesetzt werden. Dies ist ein innovativer Ansatz, der eine längere Zeit eine Progression mit wenig Toxizität erzielen kann.

Reinacher-Schick: Therapiepausen und Deeskalation.
Frage 7: Was kommt nach K-ras? Gibt es valide neue prognostische Biomarker?

Lang: Die Zukunft wird eine Vielzahl von Biomarkern bringen, die dann helfen könnten, die individuelle Therapie des KRK zu steuern.

Arnold: Ganz sicher werden weitere Marker, die den EGFRezeptor-Pathway abbilden, in die Klinik eingehen. Allerdings steht zu befürchten, dass es sich hier um weitere negative prädiktive Marker handelt, also Marker, die zeigen, welche Therapie nicht wirkt. Zuverlässige Aussagen sind hier auch aufgrund der kleinen Subkollektive schwierig; man sehe nur die aktuelle Debatte, ob BRAF nur prognostisch oder auch prädiktiv für den Nutzen einer EGFR-Therapie ist.

Aber auch hinsichtlich der antiangiogenen Therapiestrategien werden sicher in naher Zukunft noch weitere Marker gefunden werden: in erster Linie wahrscheinlich Marker, die die Therapiedauer anhand des Cytokin-Profils anzeigen. Aber auch klar prädiktive Marker, die auf die Liganden-Konzentration oder auf Rezeptorpolymorphismen zielen, dürften sich in den nächsten beiden Jahren in den Vordergrund spielen.

Reinacher-Schick: Bislang nein. BRAF ist zwar ein negativer prognostischer Marker, jedoch sollte dieser Marker ohne Möglichkeit der Therapie bei BRAF-mutierten Tumoren nicht bestimmt werden. Hinzu kommt MSI bei Niedrigrisiko-Stadium-II-Karzinomen.

\section{Teilnehmer}

Prof. Dr. med. Dirk Arnold Hubertus Wald Tumorzentrum Universitäres Cancer Center Hamburg Universitätsklinikum Hamburg-Eppendorf Martinistraße 52, 20246 Hamburg, Deutschland d.arnold@uke.de

Prof. Dr. med. Hauke Lang

Klinik und Poliklinik für Allgemein- und Abdominalchirurgie Johannes Gutenberg-Universität Mainz Langenbeckstraße 1, 55131 Mainz, Deutschland Lang@ach.klinik.uni-mainz.de

PD Dr. med. Anke Reinacher-Schick

Medizinische Universitätsklinik Knappschaftskrankenhaus, Ruhr-Universität Bochum In der Schornau 23-25, 44892 Bochum, Deutschland

Anke.Reinacher@ruhr-uni-bochum.de 